\title{
Uncountable cardinals have the same monadic $\forall 1$ positive theory over large sets
}

\author{
by
}

Athanassios Tzouvaras (Thessaloniki)

\begin{abstract}
We show that uncountable cardinals are indistinguishable by sentences of the monadic second-order language of order of the form $(\forall X) \phi(X)$ and $(\exists X) \phi(X)$, for $\phi$ positive in $X$ and containing no set-quantifiers, when the set variables range over large (= cofinal) subsets of the cardinals. This strengthens the result of Doner-MostowskiTarski [3] that $(\kappa, \in),(\lambda, \in)$ are elementarily equivalent when $\kappa, \lambda$ are uncountable. It follows that we can consistently postulate that the structures $\left(2^{\kappa},\left[2^{\kappa}\right]^{>\kappa},<\right),\left(2^{\lambda},\left[2^{\lambda}\right]^{>\lambda},<\right)$ are indistinguishable with respect to $\forall_{1}^{1}$ positive sentences. A consequence of this postulate is that $2^{\kappa}=\kappa^{+}$iff $2^{\lambda}=\lambda^{+}$for all infinite $\kappa, \lambda$. Moreover, if measurable cardinals do not exist, $\mathrm{GCH}$ is true.
\end{abstract}

1. Preliminaries. Let $\mathcal{L}=\{\prec\}$ be the first-order language of order with equality. The following is a special case of a much more general result proved in [3]:

Theorem 1.1. For any uncountable cardinals $\kappa, \lambda(\kappa,<) \equiv_{\mathcal{L}}(\lambda,<)$, where $<$ is the natural ordering of ordinals (i.e., $<=\in$ ).

Proof. The reader is warned that there is a mistake in the definition of congruence modulo $\omega^{\omega}$ given in [3, p. 51], as Professor Doner kindly informed me. The correct definition, which can be found e.g. in [2], is as follows: The ordinals $\alpha, \beta$ are congruent modulo $\omega^{\omega}$ if there are $\xi, \eta$ and $\delta<\omega^{\omega}$ such that $\alpha=\omega^{\omega} \cdot \xi+\delta, \beta=\omega^{\omega} \cdot \eta+\delta$ and either $\xi=\eta=0$ or both $\xi \neq 0$ and $\eta \neq 0$. Since for all cardinals $\kappa, \lambda>\omega, \kappa=\omega^{\omega} \cdot \kappa$ and $\lambda=\omega^{\omega} \cdot \lambda$, it follows that $\kappa, \lambda$ are congruent modulo $\omega^{\omega}$. So the claim follows from Corollary 44 of $[3]$.

Henceforth we write $(\kappa,<) \equiv(\lambda,<)$ instead of $(\kappa,<) \equiv_{\mathcal{L}}(\lambda,<)$. Let $x, y, z, \ldots$ range over the individual variables of $\mathcal{L}$. The monadic (secondorder) extension of $\mathcal{L}$, denoted by $\mathcal{L}_{\text {mon }}$, is $\mathcal{L}$ augmented with $\in$ and set variables $X, Y, Z, \ldots$ Hence $\mathcal{L}_{\text {mon }}$ has $x \in X$ as additional atoms. The stan-

2000 Mathematics Subject Classification: 03E10, 03E70.

Key words and phrases: monadic second-order language of order, positive formula. 
dard interpretations of $\mathcal{L}_{\text {mon }}$ are the structures $(A, \mathcal{P}(A),<)$, where $(A,<)$ is an ordered set and $\mathcal{P}(A)$ is the set of its subsets. The expressive power of $\mathcal{L}_{\text {mon }}$ is huge compared to that of $\mathcal{L}$. For instance it is shown in [1] that each of the structures $\left(\omega_{n},<\right), n \geq 0$, is finitely axiomatizable and categorical with respect to $\mathcal{L}_{\text {mon }}$ (under the standard interpretation). That is, for every cardinal $\omega_{n}$, the ordered set $\left(\omega_{n},<\right)$ is characterized, up to isomorphism, by a sentence of $\mathcal{L}_{\text {mon }}$.

However there are more general interpretations of $\mathcal{L}_{\text {mon }}$ besides the standard one. These are of the form $(A, \mathcal{A},<)$, where $\mathcal{A} \subset \mathcal{P}(A)$. $\mathcal{A}$ may be some class of "small" sets, i.e., an ideal of $\mathcal{P}(A)$, or, in the opposite direction, a class of "large" sets. Given cardinals $\kappa<\lambda$, let us fix the following notation:

$$
\begin{gathered}
{[\lambda]^{<\kappa}=\{X \subseteq \lambda:|X|<\kappa\}, \quad[\lambda]^{\kappa}=\{X \subseteq \lambda:|X|=\kappa\},} \\
{[\lambda]^{>\kappa}=\{X \subseteq \lambda:|X|>\kappa\}, \quad \operatorname{Cof}(\lambda)=\{X \subseteq \lambda: X \text { is cofinal in } \lambda\} .}
\end{gathered}
$$

Clearly if $\kappa$ is regular then $[\kappa]^{\kappa}=\operatorname{Cof}(\kappa)$. We often call the elements of $[\kappa]^{<\kappa}$ small subsets of $\kappa$. Accordingly we call the sets of $[\kappa]^{\kappa}$ and $\operatorname{Cof}(\kappa)$ large and cofinal, respectively. Then $X$ is co-small, i.e., $-X \in[\kappa]^{<\kappa}$, iff $X \cap Y \neq \emptyset$ for every $Y \in[\kappa]^{\kappa}$.

The aim of this paper is to strengthen Theorem 1.1 above as follows: For any uncountable $\kappa, \lambda$, the second-order structures $(\kappa$, $\operatorname{Cof}(\kappa),<)$ and $(\lambda, \operatorname{Cof}(\lambda),<)$ are indistinguishable with respect to $\forall_{1}^{1}$ and $\exists_{1}^{1}$ positive formulas (defined below).

Both positive and $\forall_{1}^{1}$ are natural classes of formulas. As an application, observe that the instance $2^{\kappa}=\kappa^{+}$of the GCH can be expressed by the truth of the $\forall_{1}^{1}$ positive formula $\forall X\left(X\right.$ is cofinal) in the structure $\left(2^{\kappa},\left[2^{\kappa}\right]^{>\kappa},<\right)$. By the main result of the paper, we can consistently postulate (see Section 4 ) that for all infinite $\kappa, \lambda$, the structures $\left(2^{\kappa},\left[2^{\kappa}\right]^{>\kappa},<\right),\left(2^{\lambda},\left[2^{\lambda}\right]^{>\lambda},<\right)$ are indistinguishable with respect to $\forall_{1}^{1}$ positive formulas.

Concerning the optimality of the result: We do not know whether $(\kappa, \operatorname{Cof}(\kappa),<)$ and $(\lambda, \operatorname{Cof}(\lambda),<)$ are indistinguishable with respect to all $\forall_{1}^{1}$ and $\exists_{1}^{1}$ formulas. Also we do not know if the structures $(\kappa, \mathcal{P}(\kappa),<)$ and $(\lambda, \mathcal{P}(\lambda),<)$ are indistinguishable either with respect to all $\forall_{1}^{1}$ and $\exists_{1}^{1}$ formulas, or with respect to positive $\forall_{1}^{1}$ and $\exists_{1}^{1}$ formulas. What we do know is that the structures $\left(\omega_{1}, \operatorname{Cof}\left(\omega_{1}\right),<\right)$ and $\left(\omega_{2}, \operatorname{Cof}\left(\omega_{2}\right),<\right)$ are distinguishable by a formula of the form $(\forall x)(\exists X) \psi$, where $\psi$ has no second-order quantifiers but is not positive. And analogously for the structures concerning $\omega_{m}, \omega_{n}$ (see the last section).

We come to the definition of positive formulas.

Definition 1.2. Let $\phi(X)$ be a formula of $\mathcal{L}_{\text {mon }}$ with at most one set variable $X$. Then $\phi(X)$ is normal if it has no set quantifiers. A normal formula $\phi$ is positive in the set variable $X$ (or just positive) if it belongs to the 
smallest class of formulas which (i) contains all formulas of $\mathcal{L}$, (ii) contains the atomic formulas $x \in X$ and (iii) is closed under the logical operations $\wedge, \vee$, and the first-order quantifiers $\exists$ and $\forall$.

The following result is due to Moschovakis [6]:

LEMma 1.3 (Moschovakis [6, p. 59]). Let $\phi(X)$ be a formula of $\mathcal{L}_{\text {mon }}$ positive in $X$. Then there is a quantifier-free and $X$-free formula $\theta(\bar{w}, u)$, where $\bar{w}=\left(w_{1}, \ldots, w_{n}\right)$, and a string of quantifiers $\bar{Q}=\left(Q_{1}, \ldots, Q_{n}\right)$ such that, for every structure $(A, \mathcal{P}(A),<)$ and every $Z \neq A$,

$$
\phi(Z) \Leftrightarrow(\bar{Q} \bar{w})(\forall u)(\theta(\bar{w}, u) \Rightarrow u \in Z)
$$

holds in $(A, \mathcal{P}(A),<)$.

In view of the above we may assume that every formula positive in $X$ has the form

$$
\phi(X) \equiv(\bar{Q} \bar{w})(\forall u)(\theta(\bar{w}, u) \Rightarrow u \in X) .
$$

We only have to make sure that $X \neq A$ when using the above form in a structure $(A,<)$. We shall refer to $(1)$ as the canonical form of $\phi$.

We shall further restrict ourselves mainly to $\forall_{1}^{1}$ positive formulas of $\mathcal{L}_{\text {mon }}$, i.e., those (equivalent to one) with prenex form $(\forall X) \phi$, where $\phi$ is positive in $X$.

2. The main theorem. From now on we shall be dealing with secondorder structures of the form $(\kappa, \operatorname{Cof}(\kappa),<)$, where $\kappa$ is an uncountable cardinal. If $\kappa$ is regular this structure is identical to $\left(\kappa,[\kappa]^{\kappa},<\right)$. Otherwise $[\kappa]^{\kappa} \subseteq \operatorname{Cof}(\kappa)$. So for brevity let us set

$$
[[\kappa]]:=(\kappa,<), \quad\left[\left[\kappa^{2}\right]\right]:=(\kappa, \operatorname{Cof}(\kappa),<) .
$$

This section contains the proof of the following:

Theorem 2.1 (Main Theorem). For all uncountable cardinals $\kappa, \lambda$,

$$
\left[\left[\kappa^{2}\right]\right] \equiv \underset{\mathrm{pos}}{\forall}\left[\left[\lambda^{2}\right]\right]
$$

i.e.,

$$
\left[\left[\kappa^{2}\right]\right] \models(\forall X) \phi(X) \Leftrightarrow\left[\left[\lambda^{2}\right]\right] \models(\forall X) \phi(X),
$$

for every positive $\phi(X)$.

Plan of the proof. To help the reader let us describe briefly the general plan of the proof. Let

$$
\phi(X) \equiv(\bar{Q} \bar{w})(\forall u)(\theta(\bar{w}, u) \Rightarrow u \in X)
$$

be a positive formula in its canonical form. Our aim is to show that $(\forall X) \phi(X)$ is absolute between $\left[\left[\kappa^{2}\right]\right]$ and $\left[\left[\lambda^{2}\right]\right]$. The proof of this fact will be split into several cases corresponding to various syntactic forms of $\phi(X)$, namely forms of $\bar{Q} \bar{w}$ and $\theta$. In each case we reduce the truth of $(\forall X) \phi(X)$ 
in $\left[\left[\kappa^{2}\right]\right]$ to that of a first-order formula in $[[\kappa]]$, which, in view of Theorem 1.1 , means that $(\forall X) \phi(X)$ is absolute between $\left[\left[\kappa^{2}\right]\right]$ and $\left[\left[\lambda^{2}\right]\right]$. Some of the cases in question are treated as Lemmas 2.2, 2.8 and 2.9 below (the rest of the lemmas provide technical tools for the treatment of $\theta$ ). After Lemma 2.9 , we concentrate on three remaining cases which are treated explicitly as Cases 1, 2 and 3 with several subcases. After exhausting them all, we give the final proof in which we simply summarize our line of thought.

To start with, given $\kappa$, it is convenient to rewrite $\left[\left[\kappa^{2}\right]\right]=\phi(X)$ as follows: We set, for every $\bar{w} \in \kappa$,

$$
R_{\theta}^{\kappa}(\bar{w})=\{u \in \kappa:[[\kappa]] \mid=\theta(\bar{w}, u)\} .
$$

Then

$$
(\forall u)(\theta(\bar{w}, u) \Rightarrow u \in X) \Leftrightarrow R_{\theta}^{\kappa}(\bar{w}) \subseteq X,
$$

so, over $\kappa$, the following identification will often be used:

$$
\phi(X) \equiv(\bar{Q} \bar{w})\left[R_{\theta}^{\kappa}(\bar{w}) \subseteq X\right] .
$$

We consider the following easy case first:

Lemma 2.2. Let $\bar{Q}$ contain no existential quantifier, i.e., $\bar{Q}=\bar{\forall}$. Then for all uncountable $\kappa, \lambda$,

$$
\left[\left[\kappa^{2}\right]\right] \models(\forall X) \phi(X) \Leftrightarrow\left[\left[\lambda^{2}\right]\right] \models(\forall X) \phi(X) .
$$

Proof. Suppose $\bar{Q}=\bar{\forall}$. Then, by (2) above,

$$
\left[\left[\kappa^{2}\right]\right] \models(\forall X) \phi(X) \Leftrightarrow\left[\left[\kappa^{2}\right]\right] \models(\forall X)(\bar{\forall} \bar{w})\left[R_{\theta}^{\kappa}(\bar{w}) \subseteq X\right] .
$$

Now it is easy to see that

$$
\left[\left[\kappa^{2}\right]\right] \models(\forall X)(\bar{\forall} \bar{w})\left[R_{\theta}^{\kappa}(\bar{w}) \subseteq X\right] \Leftrightarrow[[\kappa]] \models(\bar{\forall} \bar{w})\left[R_{\theta}^{\kappa}(\bar{w})=\emptyset\right] .
$$

Direction " $\Leftarrow$ " of $(3)$ is immediate: If $(\bar{\forall} \bar{w})\left[R_{\theta}^{\kappa}(\bar{w})=\emptyset\right]$ holds in $[[\kappa]]$, then for every cofinal $X,(\bar{\forall} \bar{w})\left[R_{\theta}^{\kappa}(\bar{w}) \subseteq X\right]$ holds in $\left[\left[\kappa^{2}\right]\right]$, hence so does $(\forall X)(\bar{\forall} \bar{w})\left[R_{\theta}^{\kappa}(\bar{w}) \subseteq X\right]$. Conversely, suppose $\left[\left[\kappa^{2}\right]\right] \models(\forall X)(\forall \bar{w})\left[R_{\theta}^{\kappa}(\bar{w}) \subseteq X\right]$. Pick any two disjoint cofinal $Z_{1}, Z_{2}$. Then for any tuple $\bar{a} \in \kappa$, we have $R_{\theta}^{\kappa}(\bar{a}) \subseteq Z_{1}$ and $R_{\theta}^{\kappa}(\bar{a}) \subseteq Z_{2}$. Since $Z_{1} \cap Z_{2}=\emptyset$, it follows that $R_{\theta}^{\kappa}(\bar{a})=\emptyset$. Since this holds for every $\bar{a} \in \kappa,[[\kappa]] \models(\bar{\forall} \bar{w})\left[R_{\theta}^{\kappa}(\bar{w})=\emptyset\right]$ and (3) is proved. Now by (3) and Theorem 1.1, for uncountable $\kappa, \lambda$ we have

$$
\begin{aligned}
{\left[\left[\kappa^{2}\right]\right] \models(\forall X) \phi(X) } & \Leftrightarrow[[\kappa]]=(\bar{\forall} \bar{w})\left[R_{\theta}^{\kappa}(\bar{w})=\emptyset\right] \\
& \Leftrightarrow[[\lambda]]=(\bar{\forall} \bar{w})\left[R_{\theta}^{\lambda}(\bar{w})=\emptyset\right] \Leftrightarrow\left[\left[\lambda^{2}\right]\right] \models(\forall X) \phi(X) .
\end{aligned}
$$

If $\bar{Q}$ in the above canonical form of $\phi(X)$ contains existential quantifiers, then a more thorough analysis of the structure of $\theta$ is needed. $\theta(\bar{w}, u)$ is a Boolean combination of atomic formulas of $\mathcal{L}=\{\prec\}$. Therefore $\theta$ is a Boolean combination of atoms $t \prec s$ and $t=s$, where $t, s \in\left\{u, w_{1}, \ldots, w_{n}\right\}$. But over a linearly ordered set the formulas $t \nprec s$ and $t \neq s$ are equivalent to $s \prec t \vee s=t$ and $t \prec s \vee s \prec t$, respectively, therefore $\theta$ is a 
$\{\vee, \wedge\}$-combination of atoms $t \prec s$ and $t=s$. So $\theta(\bar{w}, u)$ has the following disjunctive normal form:

$$
\theta(\bar{w}, u)=\bigvee_{1 \leq p \leq k} \sigma_{p}(\bar{w}, u),
$$

where each $\sigma_{p}$ is a conjunction of atoms $t \prec t, t=s$. We call $\sigma_{p}$ 's clauses. A clause is consistent if it does not contain contradictory atoms, e.g. of the form $t \prec s \wedge s \preceq t$, or $t \prec s \wedge t=s$. We may assume that each $\sigma_{p}$ is consistent (otherwise we drop it).

Definition 2.3. A clause $\sigma_{p}$ is said to be full if for any two variables $t, s$ occurring in it, $\sigma_{p}$ proves one of the formulas $t \prec s, s \prec t, t=s$. A filling of $\sigma_{p}$ is a formula $\sigma_{p}^{\prime}$ which is consistent, full, contains the same variables as $\sigma_{p}$, and $\sigma_{p}^{\prime} \vdash \sigma_{p}$. (That is, $\sigma_{p}^{\prime}$ contains some extra atoms that guarantee completeness.)

If $\sigma_{p}$ is a full clause containing the variables $t_{1}, \ldots, t_{m} \in\left\{u, w_{1}, \ldots, w_{n}\right\}$, then it is clear that (by renaming $t_{i}$ if necessary) $\sigma_{p}$ entails (and is equivalent to) a total ordering of $t_{i}$ of the form $t_{1} \preceq t_{2} \preceq \cdots \preceq t_{m}$ (where of course $\preceq$ means $\prec$ or $=$ ). More precisely, the preceding relation is written $t_{1} R t_{2} R \cdots R t_{m}$, where $R$ is either $\prec$ or $=$. Thus we have shown clause (i) of the following (clause (ii) is straightforward).

LEMMA 2.4. (i) If $\sigma_{p}$ is complete, then it is equivalent to a conjunction of the form

$$
t_{1} R t_{2} R \cdots R t_{m},
$$

where $R$ is either $\prec$ or $=$ and all $t_{i}$ are distinct variables from among $\left\{u, w_{1}, \ldots, w_{n}\right\}$.

(ii) Every (consistent) $\sigma_{p}$ has a filling, and if $\sigma_{p i}^{\prime}, i \leq l$, are all the distinct completions of $\sigma_{p}$, then $\sigma_{p}=\bigvee_{i \leq l} \sigma_{p i}$.

In view of 2.4(ii), we may assume that every clause $\sigma_{p}$ in the analysis (4) of $\theta$ is full, hence, by 2.4(i), it is of the form

$$
t_{1} R t_{2} R \cdots R t_{m}
$$

$\sigma_{p}$ is said to be a $u$-clause if it contains the variable $u$. Otherwise it is called $u$-free. If $\delta$ denotes the disjunction of all $u$-free clauses, then $\theta$ is written

$$
\theta(\bar{w}, u) \equiv \bigvee_{1 \leq p \leq k} \sigma_{p}(\bar{w}, u) \vee \delta(\bar{w}),
$$

where each $\sigma_{p}$ is a $u$-clause. Further, each $u$-clause $\sigma_{p}$ consists of $u$-atoms and $u$-free atoms. Writing $\zeta_{p}$ and $\eta_{p}$ for the conjunction of $u$-atoms and $u$-free atoms of $\sigma_{p}$ respectively, we have

$$
\sigma_{p}(\bar{w}, u) \equiv \zeta_{p}(\bar{w}, u) \wedge \eta_{p}(\bar{w}) .
$$


A $u$-clause $\sigma_{p}(\bar{w}, u)$ is said to be equational in $u$ (or just equational) if it contains an identity $u=t$.

Now if $\sigma_{p}$ is equational we may assume that $\zeta_{p}$ consists of a single equation $u=t$, i.e., $\zeta_{p} \equiv\left(u=t_{k_{p}}\right)$. Indeed, first we may assume that $\sigma_{p}$ contains only one $u$-equation. Otherwise we shall have the sequence

$$
t_{1} R t_{2} R \cdots t_{i}=u=t_{j} R \cdots R t_{m}
$$

which can be simply rewritten as

$$
t_{1} R t_{2} R \cdots R t_{i}=t_{j}=u R \cdots R t_{m},
$$

or

$$
t_{1} R t_{2} R \cdots R u=t_{i}=t_{j} R \cdots R t_{m} .
$$

If $\sigma_{p}$ contains moreover a $u$-literal which is not an equation, say $u=t_{1} \wedge$ $u \prec t_{2}$, then $\left(u=t_{1} \wedge u \prec t_{2}\right) \equiv\left(u=t_{1} \wedge t_{1} \prec t_{2}\right)$. So $\zeta_{p}$ can be taken to be a single equation and thus an equational $\sigma_{p}$ has the form

$$
\sigma_{p} \equiv\left(u=t_{k_{p}}\right) \wedge \eta_{p}
$$

On the other hand, if a $u$-clause $\sigma_{p}$ is non-equational, then $u$ occurs only in inequalities, so $\sigma_{p}$ will be of the form either

$$
t_{1} R t_{2} R \cdots R t_{i} \prec u \prec t_{j} R \cdots R t_{m},
$$

or

$$
u \prec t_{1} R t_{2} R \cdots R t_{m}
$$

or

$$
t_{1} R t_{2} R \cdots R t_{m} \prec u .
$$

Therefore every non-equational $\sigma_{p}$ has the form

$$
\sigma_{p} \equiv\left(t_{i_{p}} \prec u \prec t_{j_{p}}\right) \wedge \eta_{p}
$$

where one of the $t_{i_{p}}, t_{j_{p}}$ may be missing. For every equational $\sigma_{p}(\bar{w}, u)$ and for a given cardinal $\kappa$, let

$$
E_{p}^{\kappa}(\bar{w})=\left\{u:[[\kappa]] \models \sigma_{p}(\bar{w}, u)\right\}
$$

( $E$ for "equation"). Similarly for non-equational $\sigma_{p}(\bar{w}, u)$ and $\kappa$, let

( $I$ for "interval"). Finally, let

$$
I_{p}^{\kappa}(\bar{w})=\left\{u:[[\kappa]]=\sigma_{p}(\bar{w}, u)\right\}
$$

$$
D^{\kappa}(\bar{w})=\{u:[[\kappa]] \models \delta(\bar{w})\} .
$$

By the above analysis we get the following immediately:

Lemma 2.5. (i) If $\sigma_{p}$ is equational, then $E_{p}^{\kappa}(\bar{w})$ is a singleton or $\emptyset$, namely,

$$
E_{p}^{\kappa}(\bar{w})=\left\{u: u=t_{k_{p}} \wedge \eta_{p}(\bar{w})\right\},
$$

where $t_{k_{p}} \in\left\{w_{1}, \ldots, w_{n}\right\}$. 
(ii) If $\sigma_{p}$ is non-equational, then $I_{p}^{\kappa}(\bar{w})$ is an interval (maybe empty), namely

$$
I_{p}^{\kappa}(\bar{w})=\left\{u: t_{i_{p}} \prec u \prec t_{j_{p}} \wedge \eta_{p}(\bar{w})\right\},
$$

where $t_{i_{p}}, t_{j_{p}} \in\left\{w_{1}, \ldots, w_{n}\right\}$ and one of the $t_{i_{p}}, t_{j_{p}}$ may be missing.

(iii) $D^{\kappa}(\bar{w})$ is either $\kappa$ or $\emptyset$.

(iv) $R_{\theta}^{\kappa}(\bar{w})=\bigcup_{1 \leq p \leq r} E_{p}^{\kappa}(\bar{w}) \cup \bigcup_{r<p \leq k} I_{p}^{\kappa}(\bar{w}) \cup D^{\kappa}(\bar{w})$ for some $r \leq k$.

LEMMA 2.6. Let $\left[\left[\kappa^{2}\right]\right] \models(\forall X) \phi(X)$. Then

$\left[\left[\kappa^{2}\right]\right] \models(\forall X)(\bar{Q} \bar{w})\left[R_{\theta}^{\kappa}(\bar{w})\right.$ contains no non-trivial interval $\left.\wedge R_{\theta}^{\kappa}(\bar{w}) \subseteq X\right]$.

Proof. Note first that " $R_{\theta}^{\kappa}(\bar{w})$ contains no non-trivial interval" is the informal formulation of a formula of $\mathcal{L}$. Assume the contrary, i.e., that there is a cofinal set $Z$ such that

$$
\left[\left[\kappa^{2}\right]\right] \models\left(\bar{Q}^{\prime} \bar{w}\right)\left[R_{\theta}^{\kappa}(\bar{w}) \text { contains a non-trivial interval } \vee R_{\theta}^{\kappa}(\bar{w}) \nsubseteq Z\right],
$$

where $\bar{Q}^{\prime}$ is the dual string of $\bar{Q}$. But then we can get a cofinal $Y \subseteq Z$ that contains no non-trivial interval (e.g. the limit points of the ordering $(Z,<)$ ). So, clearly, the sentence " $R_{\theta}^{\kappa}(\bar{w})$ contains a non-trivial interval" implies $R_{\theta}^{\kappa}(\bar{w}) \nsubseteq Y$ and also $R_{\theta}^{\kappa}(\bar{w}) \nsubseteq Z$ implies $R_{\theta}^{\kappa}(\bar{w}) \nsubseteq Y$. Therefore the above disjunction implies

$$
\left[\left[\kappa^{2}\right]\right]=\left(\bar{Q}^{\prime} \bar{w}\right)\left[R_{\theta}^{\kappa}(\bar{w}) \nsubseteq Y\right] .
$$

It follows that $\left[\left[\kappa^{2}\right]\right]=(\exists X)\left(\bar{Q}^{\prime} \bar{w}\right)\left[R_{\theta}^{\kappa}(\bar{w}) \nsubseteq X\right]$. Since

$$
\phi(X) \equiv(\bar{Q} \bar{w})\left[R_{\theta}^{\kappa}(\bar{w}) \subseteq X\right],
$$

we get $\left[\left[\kappa^{2}\right]\right] \models(\exists X) \neg \phi(X)$, which contradicts our assumption.

LemMA 2.7. Let $\phi(X) \equiv(\bar{Q} \bar{w})(\forall u)(\theta(\bar{w}, u) \Rightarrow u \in X)$, and let $\left[\left[\kappa^{2}\right]\right] \models$ $(\forall X) \phi(X)$. Then there is a quantifier free $\theta^{*}(\bar{w}, \bar{z}, u)$ and a string of quantifiers $\bar{Q}^{*}$ such that if $\phi^{*}(X) \equiv\left(\bar{Q}^{*} \overline{w z}\right)(\forall u)\left(\theta^{*}(\bar{w}, u) \Rightarrow u \in X\right)$, then $\phi(X)$ and $\phi^{*}(X)$ are equivalent over $\left[\left[\kappa^{2}\right]\right]$ and

$\left[\left[\kappa^{2}\right]\right] \models(\forall X)\left(\bar{Q}^{*} \overline{w z}\right)\left[R_{\theta^{*}}^{\kappa}(\bar{w}, \bar{z})\right.$ contains no non-trivial interval

$$
\left.\wedge R_{\theta *}^{\kappa}(\bar{w}, \bar{z}) \subseteq X\right]
$$

and $\theta^{*}(\bar{w}, \bar{z}, u)=\bigvee_{q} \tau_{q}(\bar{w}, \bar{z}, u)$, where all $\tau_{q}$ are equational.

Proof. By the assumption and Lemma 2.6,

$$
\begin{aligned}
{\left[\left[\kappa^{2}\right]\right]=(\forall X)(\bar{Q} \bar{w})\left[R_{\theta}^{\kappa}(\bar{w})\right. \text { contains no non-trivial interval }} & \\
& \left.\wedge R_{\theta}^{\kappa}(\bar{w}) \subseteq X\right] .
\end{aligned}
$$

Recall from Lemma 2.5(iv) that $R_{\theta}^{\kappa}(\bar{w})$ can be written in the form

$$
R_{\theta}^{\kappa}(\bar{w})=\bigcup_{1 \leq p \leq r} E_{p}^{\kappa}(\bar{w}) \cup \bigcup_{r<p \leq k} I_{p}^{\kappa}(\bar{w}) \cup D^{\kappa}(\bar{w}) .
$$

Since the set $D^{\kappa}(\bar{w})$ is either $\emptyset$ or $\kappa$, it follows from $(8)$ that $D^{\kappa}(\bar{w})=\emptyset$ (otherwise $D^{\kappa}(\bar{w})=R_{\theta}^{\kappa}(\bar{w})=\kappa$, which contains non-trivial intervals). 
Therefore

$$
R_{\theta}^{\kappa}(\bar{w})=\bigcup_{1 \leq p \leq r} E_{p}^{\kappa}(\bar{w}) \cup \bigcup_{r<p \leq k} I_{p}^{\kappa}(\bar{w}) .
$$

Let $I_{p}^{\kappa}$ be some of the intervals contained above, defined by a nonequational clause (7). By (8),

$$
[[\kappa]] \models(\bar{Q} \bar{w})\left(I_{p}^{\kappa}=\emptyset \text { or a singleton }\right) .
$$

But the fact that $t_{i_{p}} \prec u \prec t_{j_{p}} \wedge \eta_{p}$ defines at most a singleton implies that the latter formula can be equivalently replaced by

$$
\left(t_{i_{p}}^{\prime}=u \wedge u^{\prime}=t_{j_{p}}^{\prime}\right) \wedge \eta_{p}
$$

where $t^{\prime}=s$ abbreviates " $s$ is the immediate successor of $t$ ". The above formula is analytically written as

$$
\left(\exists z_{1}\right)\left[t_{i_{p}} \prec z_{1} \prec t_{j_{p}} \wedge\left(\forall z_{2}\right)\left(z_{2} \preceq t_{i_{p}} \vee z_{2}=u \vee t_{j_{p}} \preceq z_{2}\right)\right] \wedge \eta_{p} .
$$

But (9) is equational in $u$, at the cost that it contains some extra bound variables.

Concerning intervals of the form $\left(t_{i_{p}}, \infty\right)$, or $\left[0, t_{j_{p}}\right)$, in view of $(8)$, if $\sigma_{p}$ is of the form $\left(t_{i_{p}} \prec u\right) \wedge \eta_{p}$, then the defined interval must always be $\emptyset$, therefore we can replace $\left(t_{i_{p}} \prec u\right) \wedge \eta_{p}$ by $\perp$. If $\sigma_{p}$ is of the form $\left(u \prec t_{j_{p}}\right) \wedge \eta_{p}$, then, in order for $\left[0, t_{j_{p}}\right)$ to be trivial, we must have either $\neg \eta$, or $t_{j_{p}}=0$, or $t_{j_{p}}=0^{\prime}$. Therefore, $\left(u \prec t_{j_{p}}\right) \wedge \eta_{p}$ can be replaced by the formula $\left(t_{j_{p}}=0 \vee t_{j_{p}}=0^{\prime}\right) \wedge \eta$.

Now if we replace each non-equational clause $\sigma_{p}$ by a formula of this kind and put the resulting formula in prenex normal form, we shall get a formula $\theta^{*}(\bar{w}, \bar{z}, u)$ whose disjunctive normal form contains only $u$-equational clauses. Moreover, if $\phi^{*}(X)$ is the positive formula resulting from $\theta^{*}$ in the obvious way, then $\phi(X)$ and $\phi^{*}(X)$ are equivalent over $\left[\left[\kappa^{2}\right]\right]$ and

$$
\begin{aligned}
{\left[\left[\kappa^{2}\right]\right] \models(\forall X)\left(\bar{Q}^{*} \overline{w z}\right)\left[R_{\theta^{*}}^{\kappa}(\bar{w}, \bar{z})\right. \text { contains no non-trivial interval }} \\
\left.\wedge R_{\theta^{*}}^{\kappa}(\bar{w}, \bar{z}) \subseteq X\right] .
\end{aligned}
$$

Let $\bar{Q}$ contain existential quantifiers and let $\left[\left[\kappa^{2}\right]\right] \models(\forall X) \phi(X)$. By Lemma 2.7, we may assume that if $\theta=\bigvee_{p} \sigma_{p}$, then all $\sigma_{p}$ are equational clauses. This simplifies things considerably and we now turn to examining the form of the string $\bar{Q}$. Without essential loss of generality, and for the sake of illustrating the argument, assume that $\bar{Q}$ consists of simple alternations of $\forall$ and $\exists$, starting with $\forall$. Let henceforth $\bar{w}=\left(w_{1}, \ldots, w_{n}\right)$ denote the string of variables bound by $\forall$ and let $\bar{v}=\left(v_{1}, \ldots, v_{n}\right)$ denote the string of variables bound by $\exists$. So instead of writing $\bar{Q} \bar{w}$ we henceforth write $\bar{Q} \overline{w v}$, where

$$
\bar{Q} \overline{w v}=\left(\forall w_{1}\right)\left(\exists v_{1}\right)\left(\forall w_{2}\right) \cdots\left(\forall w_{n}\right)\left(\exists v_{n}\right) .
$$


Then $\left[\left[\kappa^{2}\right]\right] \models(\forall X) \phi(X)$ is written as

$$
\left[\left[\kappa^{2}\right]\right] \models(\forall X)\left(\forall w_{1}\right)\left(\exists v_{1}\right) \cdots\left(\forall w_{n}\right)\left(\exists v_{n}\right)\left[R_{\theta}^{\kappa}(\bar{w}, \bar{v}) \subseteq X\right],
$$

where $R_{\theta}^{\kappa}(\bar{w}, \bar{v})$ is a finite union of singletons $\left\{w_{i}\right\}$ or $\left\{v_{j}\right\}$. We divide the equational clauses of $\theta$ into $(u=w)$-clauses, if the $u$-equation contained is of the form $u=w_{i}$ (independent variable), and ( $u=v$ )-clauses, if the $u$ equation contained is of the form $u=v_{j}$ (dependent variable). Also we refer to the independent and dependent variables as $w$-variables and $v$-variables respectively.

Lemma 2.8. If $\theta$ consists of $(u=v)$-clauses only, then $\left[\left[\kappa^{2}\right]\right] \models(\forall X) \phi(X)$, hence for all $\kappa, \lambda$,

$$
\left[\left[\kappa^{2}\right]\right] \models(\forall X) \phi(X) \Leftrightarrow\left[\left[\lambda^{2}\right]\right] \models(\forall X) \phi(X) .
$$

Proof. Let $\theta \equiv \bigvee_{p} \sigma_{p}$, where all $\sigma_{p}$ are $(u=v)$-clauses. We have to show that

$$
\left[\left[\kappa^{2}\right]\right] \models(\forall X)(\bar{Q} \overline{w v})(\forall u)\left(\bigvee_{p} \sigma_{p} \Rightarrow u \in X\right) .
$$

Now given any cofinal $X$, it suffices to pick an arbitrary $\bar{v} \in X$ no matter what the choice of $\bar{w}$ is. Then each $\sigma_{p}$ will define either a singleton $\left\{v_{j}\right\} \subseteq X$, if $\eta_{p}$ is satisfied, or $\emptyset$ otherwise. Thus in any case $R_{\theta}^{\kappa}(\bar{w}, \bar{v}) \subseteq X$, and we are done.

LEMMA 2.9. If some $(u=w)$-clause contains only $w$-variables, then for any $\kappa,\left[\left[\kappa^{2}\right]\right] \not \forall(\forall X) \phi(X)$, hence for all $\kappa, \lambda$,

$$
\left[\left[\kappa^{2}\right]\right] \models(\forall X) \phi(X) \Leftrightarrow\left[\left[\lambda^{2}\right]\right] \models(\forall X) \phi(X) .
$$

Proof. Let $\sigma_{p}$ be a clause containing only variables of type $w_{i}$. Then it has the form

$$
w_{i_{1}} R \cdots R u=w_{i_{p}} R \cdots R w_{i_{l}},
$$

where $R$ is either $\prec$ or $=$. Let $\bar{Q}^{\prime}$ be the dual string of the string $\bar{Q}$ of $\phi$. Then $\bar{Q}^{\prime}$ contains $\left(\exists w_{i_{1}}\right) \cdots\left(\exists w_{i_{p}}\right) \cdots\left(\exists w_{i_{l}}\right)$ as a substring. Moreover, since the other variables of $(\bar{w}, \bar{v})$ do not affect $\sigma_{p}$,

$$
[[\kappa]] \models\left(\bar{Q}^{\prime} \overline{w v}\right)(\exists u) \sigma_{p}
$$

is equivalent to

$$
[[\kappa]] \models\left(\exists w_{i_{1}}\right) \cdots\left(\exists w_{i_{p}}\right) \cdots\left(\exists w_{i_{l}}\right)(\exists u)\left[w_{i_{1}} R \cdots R u=w_{i_{p}} R \cdots R w_{i_{l}}\right] .
$$

Now it is clear that, whatever the particular form of the clause

$$
w_{i_{1}} R \cdots R u=w_{i_{p}} R \cdots R w_{i_{l}}
$$

would be, there is a cofinal set $Z$ such that

$$
\begin{aligned}
{[[\kappa]] \models\left(\exists w_{i_{1}}\right) \cdots\left(\exists w_{i_{p}}\right) } & \cdots\left(\exists w_{i_{l}}\right)(\exists u) \\
& {\left[\left(w_{i_{1}} R \cdots R u=w_{i_{p}} R \cdots R w_{i_{l}}\right) \wedge u \notin Z\right] . }
\end{aligned}
$$


Equivalently,

$$
[[\kappa]] \models\left(\bar{Q}^{\prime} \overline{w v}\right)(\exists u)\left(\sigma_{p} \wedge u \notin Z\right) .
$$

Therefore

$$
[[\kappa]] \models\left(\bar{Q}^{\prime} \overline{w v}\right)\left(R_{\theta}^{\kappa}(\bar{w}, \bar{v}) \not \subset Z\right),
$$

whence $\left[\left[\kappa^{2}\right]\right] \not \models(\forall X) \phi(X)$.

In view of Lemmas 2.8 and 2.9, where the truth of $(\forall X) \phi(X)$ in every $\left[\left[\kappa^{2}\right]\right]$ is settled under the conditions stated, we now assume that these conditions fail, i.e., that $\theta$ contains $(u=w)$-clauses and every $(u=w)$-clause contains $v$-variables. Then we have to examine three main cases, where Cases 1 and 2 contain several subcases, sub-subcases etc. We call them all "Cases" and enumerate them by sequences of numbers. For instance, 1.2.1 and 1.2.2 are subcases of 1.2. We mark a case by $\rightarrow$ if it is a subcase of the previous one, by $\downarrow$ if it is a case of equal depth as the previous one, and by $\leftarrow$ if we return to a case of smaller depth than the previous one. The cases concern either syntactic properties of the clauses of $\theta$ or the truth in $[[\kappa]]$ of some (first-order) subformula of $\theta$. It turns out that these characteristics fully determine the truth or falsehood of $(\forall X) \phi(X)$ in $\left[\left[\kappa^{2}\right]\right]$. Therefore, in view of Theorem 1.1, the same syntactic and semantic conditions determine the truth or falsehood, respectively, of $(\forall X) \phi(X)$ in $\left[\left[\lambda^{2}\right]\right]$, for any uncountable cardinal $\lambda$. So, roughly, in the cases below we reduce the truth of the second-order formula $(\forall X) \phi(X)$ to the truth of some first-order formula plus some syntactic conditions.

CASE 1 . There are $w$-clauses in which all $v$-variables precede all $w$ variables (in the enumeration of variables given in (10)), i.e., for all variables $w_{i}$ and $v_{j}$ occurring in these clauses, we have $j<i$. Since every $v$-variable is bound by $\exists$ and every $w$-variable is bound by $\forall$, it follows that every value of the $v$-variables of the clause is independent of any value of a $w$-variable contained there.

$\rightarrow$ CASE 1.1. Suppose there is a $w$-clause $\sigma_{p} \equiv\left(u=w_{i}\right) \wedge \eta_{p}$ containing a unique $v$-variable, say $v_{j}$.

$\rightarrow$ CASE 1.1.1. $\sigma_{p}$ contains formulas of the form $w_{k} \prec v_{j}$, and specifically let $\sigma_{p}$ define $m$ elements below $v_{j}$, say by the inequalities $w_{i_{1}} \prec w_{i_{2}} \prec$ $\cdots \prec w_{i_{m}} \prec v_{j}$.

$\rightarrow$ CASE 1.1.1.1. There is no clause containing $u=v_{j}$. Then, choosing $v_{j}=0$, we falsify $\sigma_{p}$ for any choice of the independent variables, so $\left[\left[\kappa^{2}\right]\right] \models$ $(\forall X) \phi(X)$.

$\downarrow$ CASE 1.1.1.2. There are clauses containing $u=v_{j}$; let us enumerate them as $\sigma_{p_{k}} \equiv\left(u=v_{j}\right) \wedge \eta_{p_{k}}$ for $k \leq s$. 
$\rightarrow$ CASE 1.1.1.2.1. $[[\kappa]]=\left(\bar{Q}^{\prime} \overline{w v}\right)\left(\bigvee_{k \leq s} \eta_{p_{k}}\right)$, where in the string $\bar{Q}^{\prime} \overline{w v}$, we have $\left(\forall v_{j} \in\{0, \ldots, m\}\right)$ instead of $\left(\forall v_{j}\right)$. Then $\left[\left[\kappa^{2}\right]\right] \models(\exists X) \neg \phi(X)$.

Proof. Indeed, recall from the above that $w_{i_{1}} \prec w_{i_{2}} \prec \cdots \prec w_{i_{m}} \prec v_{j}$, and $j<i_{1}, \ldots, i_{m}$, so in order to show that there is $Z$ such that

$$
\left[\left[\kappa^{2}\right]\right] \models\left(\bar{Q}^{\prime}\right)\left[R_{\theta}^{\kappa}(\bar{w}, \bar{v}) \nsubseteq Z\right],
$$

it suffices to show that there is $Z$ such that

$$
\begin{aligned}
{\left[\left[\kappa^{2}\right]\right] \models } & \left(\forall v_{j}\right)\left(\exists w_{i_{1}}\right) \cdots\left(\exists w_{i_{m}}\right)(\exists u) \\
& {\left[\left(w_{i_{1}} \prec \cdots \prec w_{i_{m}} \prec v_{j} \cdots u=w_{i} \cdots \vee \bigvee_{k \leq s} \eta_{p_{k}}(\bar{w}, \bar{v})\right) \wedge u \notin Z\right] . }
\end{aligned}
$$

Take $Z$ to be a cofinal set such that $\{0, \ldots, m\} \cap Z=\emptyset$ and $-Z$ is cofinal. Then it is easy to check that the preceding formula holds in $\left[\left[\kappa^{2}\right]\right]$.

$\downarrow$ CASE 1.1.1.2.2. $[[\kappa]] \not \models\left(\bar{Q}^{\prime} \overline{w v}\right) \bigvee_{k \leq s} \eta_{p_{k}}$, where in the string $\bar{Q}^{\prime} \overline{w v}$, we have $\left(\forall v_{j} \in\{0, \ldots, m\}\right)$ instead of $\left(\forall v_{j}\right)$. That is, $[[\kappa]] \models(\bar{Q} \overline{w v})\left(\bigwedge_{k \leq s} \neg \eta_{p_{k}}\right)$, where in the string $\bar{Q} \overline{w v}$, we have $\left(\exists v_{j} \in\{0, \ldots, m\}\right)$ instead of $\left(\exists v_{j}\right)$. Then, choosing $v_{i} \in\{0, \ldots, m\}$, we simultaneously falsify $w_{i_{1}} \prec w_{i_{2}} \prec \cdots \prec w_{i_{m}} \prec$ $v_{j} \cdots u=w_{i} \cdots$ and all $\sigma_{p_{k}}$. Hence $(\forall X) \phi(X)$ holds in $\left[\left[\kappa^{2}\right]\right]$.

$\leftarrow$ CASE 1.1.2. $\sigma_{p}$ contains no formula of the form $w_{k} \prec v_{j}$, i.e., $\sigma_{p}$ is of the form

$$
v_{j} R w_{k_{1}} R \cdots u=w_{i} R \cdots R w_{k_{l}} .
$$

Then $\left[\left[\kappa^{2}\right]\right] \models(\exists X) \neg \phi(X)$.

Proof. Taking a cofinal set $Z$ whose complement is also cofinal, we have

$$
\begin{array}{r}
{\left[\left[\kappa^{2}\right]\right] \models\left(\forall v_{j}\right)\left(\exists w_{k_{1}}\right) \cdots\left(\exists w_{k_{l}}\right)(\exists u)} \\
\quad\left[\left(v_{j} R w_{k_{1}} R \cdots R u=w_{i} R \cdots R w_{k_{l}}\right) \wedge u \notin Z\right] .
\end{array}
$$

Consequently,

$$
\left[\left[\kappa^{2}\right]\right] \models\left(\bar{Q}^{\prime}\right)\left[R_{\theta}^{\kappa}(\bar{w}, \bar{v}) \not Z Z\right]
$$

i.e., $\left[\left[\kappa^{2}\right]\right] \mid=(\exists X) \neg \phi(X)$.

$\leftarrow$ CASE 1.2. There is a $(u=w)$-clause $\sigma_{p} \equiv\left(u=w_{i} \wedge \eta_{p}\right)$ containing at least two $v$-variables. Let $v_{j_{1}}, \ldots, v_{j_{t}}$ be the list of all $v$-variables of $\sigma_{p}$. We divide the $(u=w)$-clauses into two groups: (a) those defining a two-end interval with respect to $u$, i.e. $\sigma_{p}$ contains a subformula of the form $v_{j_{a}} \preceq u=$ $w_{i} \preceq v_{j_{b}}$, and (b) those defining a one-end interval, i.e. in which all $v_{j_{a}}$ are strictly before or after the equation $u=w_{i}$. We denote the clauses of the first kind by $\sigma_{p}^{2}$ and of the second kind by $\sigma_{r}^{1}$, and similarly their corresponding subformulas by $\eta_{p}^{2}$ and $\eta_{r}^{1}$ respectively. Further, let $\sigma_{q} \equiv\left(u=v_{j_{q}} \wedge \eta_{q}\right)$ be the clauses of $\theta$ (if any) containing an $(u=v)$-equation for the above mentioned variables $v_{j_{1}}, \ldots, v_{j_{t}}$. 
So the relevant subformula of $\phi(X)$ is written as

$$
\begin{aligned}
\left(\exists v_{j_{1}}\right) \cdots\left(\exists v_{j_{t}}\right)\left(\forall w_{i_{1}}\right) \cdots( & \left.\forall w_{i_{s}}\right)(\forall u) \\
& {\left[\left(\bigvee_{p} \sigma_{p}^{2} \vee \bigvee_{r} \sigma_{r}^{1} \vee \bigvee_{q} \sigma_{q}\right) \Rightarrow u \in X\right] . }
\end{aligned}
$$

$\rightarrow$ CASE 1.2.1. In (11) above there are no formulas of type $\sigma_{r}^{1}$. Then $\left[\left[\kappa^{2}\right]\right] \models(\forall X) \phi(X)$.

Proof. We have to show that

$$
\left[\left[\kappa^{2}\right]\right] \models(\forall X)\left(\exists v_{j_{1}}\right) \cdots\left(\exists v_{j_{t}}\right)\left(\forall w_{i_{1}}\right) \cdots\left(\forall w_{i_{s}}\right)(\forall u)\left[\left(\bigvee_{p} \sigma_{p}^{2} \vee \bigvee_{q} \sigma_{q}\right) \Rightarrow u \in X\right]
$$

Each of the formulas $\sigma_{p}^{2}$ contains a pair of variables $v_{j_{a}}, v_{j_{b}}$ that bound the equation $u=w_{i}$ from above and below. So given $X$ it suffices to choose $v_{j_{a}}=v_{j_{b}} \in X$. Then the intervals they define are null, independently of the choice of $w$-variables. Moreover if this choice satisfies $\eta_{q}$, then $\sigma_{q}$ defines a singleton whose element belongs to $X$. Otherwise $\sigma_{q}$ defines $\emptyset$ and we are done. So the above formula holds. Observe also that the $v_{j_{a}}, v_{j_{b}}$ can be chosen arbitrarily high.

$\downarrow$ CASE 1.2.2. In (11) above there are no formulas of type $\sigma_{r}^{2}$. Then $\left[\left[\kappa^{2}\right]\right] \models(\exists X) \neg \phi(X)$.

Proof. We have to show that

$$
\left[\left[\kappa^{2}\right]\right] \models(\exists X)\left(\forall v_{j_{1}}\right) \cdots\left(\forall v_{j_{t}}\right)\left(\exists w_{i_{1}}\right) \cdots\left(\exists w_{i_{s}}\right)(\exists u)\left[\left(\bigvee_{r} \sigma_{r}^{1} \vee \bigvee_{q} \sigma_{q}\right) \wedge u \notin X\right]
$$

Now in every formula $\sigma_{r}$, the equation $u=w_{i}$ is to the left or right of all $v$-variables, so the situation is quite similar to that of Case 1.1.

$\downarrow$ CASE 1.2.3. In (11) we have formulas of both types $\sigma_{r}^{1}$ and $\sigma_{r}^{2}$. Then we distinguish two subcases.

$\rightarrow$ CASE 1.2.3.1.

$$
[[\kappa]]=\left(\exists v_{j_{1}}\right) \cdots\left(\exists v_{j_{t}}\right)\left(\forall w_{i_{1}}\right) \cdots\left(\forall w_{i_{s}}\right)\left[\bigvee_{p} \eta_{p}^{2} \vee\left(\bigwedge_{r} \neg \eta_{r}^{1} \wedge \bigwedge_{q} \neg \eta_{q}\right)\right] .
$$

Then $\left[\left[\kappa^{2}\right]\right] \models(\forall X) \phi(X)$.

Proof. By assumption there is a valuation of the $v$-variables that makes all $\eta_{r}^{1}$ formulas defining one-end intervals false. So $\left[\left[\kappa^{2}\right]\right] \models(\forall X) \phi(X)$ is shown by the argument of Case 1.2.1 above.

$\downarrow$ CASE 1.2.3.2.

$$
[[\kappa]] \equiv\left(\forall v_{j_{1}}\right) \cdots\left(\forall v_{j_{t}}\right)\left(\exists w_{i_{1}}\right) \cdots\left(\exists w_{i_{s}}\right)\left[\bigwedge_{p} \neg \eta_{p}^{2} \wedge\left(\bigvee_{r} \eta_{r}^{1} \vee \bigvee_{q} \eta_{q}\right)\right]
$$

Then $\left[\left[\kappa^{2}\right]\right] \models(\exists X) \neg \phi(X)$. 
Proof. By assumption for every valuation of the $v$-variables there is a valuation of the $w$-variables that makes all $\eta_{r}^{2}$ formulas defining two-end intervals false. So $\left[\left[\kappa^{2}\right]\right] \models(\exists X) \neg \phi(X)$ is shown by the argument of Case 1.2.2 above.

This exhausts the examination of Case 1 . It is important to note that whenever in the above subcases $\left[\left[\kappa^{2}\right]\right] \models(\exists X) \neg \phi(X)$, then for every $X$, we can choose each $v_{j}$ arbitrarily high in $X$, unless the equation $u=v_{i}$ does not occur in $\theta$, or the choice of $v_{j}$ falsifies simultaneously all clauses containing $u=v_{j}$.

$\leftarrow$ CASE 2. There is a $\left(u=w_{i}\right)$-clause $\sigma_{p}$ containing a formula $w_{k} \prec v_{j}$ with $k \leq j$.

$\rightarrow$ CASE 2.1. $\theta$ does not contain any $\left(u=v_{j}\right)$-clause. Then $\left[\left[\kappa^{2}\right]\right] \models$ $(\forall X) \phi(X)$.

Proof. The relevant subformula of $\phi$ is

$$
\left(\forall w_{k}\right)\left(\exists v_{j}\right)(\forall u)\left[\left(u=w_{i} \wedge w_{k} \prec v_{j}\right) \Rightarrow u \in X\right] .
$$

Now since $\left(u=v_{j}\right)$ does not occur in $\theta$, given any $X$ and $w_{k}$, we can pick, say, $v_{j}=w_{k}$, falsifying thus the hypothesis of the above implication, i.e., $\sigma_{p}$, and so $\left[\left[\kappa^{2}\right]\right]=(\forall X) \phi(X)$.

$\downarrow$ CASE 2.2. $\theta$ contains $\left(u=v_{j}\right)$-clauses and let $\left(\sigma_{q}\right)_{q}$ be an enumeration of them. Let $\eta_{p}, \eta_{q}$ be the corresponding subformulas of them.

$\rightarrow$ CASE 2.2.1.

$$
[[\kappa]] \models\left(\forall w_{k}\right)\left(\exists v_{j}\right)\left[\neg \eta_{p} \wedge \bigwedge_{q} \neg \eta_{q}\right] .
$$

Then clearly

$$
\left[\left[\kappa^{2}\right]\right] \models(\forall X)\left(\forall w_{k}\right)\left(\exists v_{j}\right)(\forall u)\left[\left(\neg \eta_{p} \wedge \bigwedge_{q} \neg \eta_{q}\right) \Rightarrow u \in X\right],
$$

that is, $\left[\left[\kappa^{2}\right]\right] \models(\forall X) \phi(X)$.

$\downarrow$ CASE 2.2.2.

$$
[[\kappa]]=\left(\exists w_{k}\right)\left(\forall v_{j}\right)\left[\eta_{p} \vee \bigvee_{q} \eta_{q}\right]
$$

We claim that $\left[\left[\kappa^{2}\right]\right] \models(\exists X) \neg \phi(X)$.

Proof. We examine the relative positions of $w_{i}, w_{k}, v_{j}$ in $\sigma_{p}$. They are: (a) $u=w_{i} \preceq w_{k} \prec v_{j}$, (b) $w_{k} \prec u=w_{i} \preceq v_{j}$, or (c) $w_{k} \prec v_{j} \preceq u=w_{i}$.

(a) Let $u=w_{i} \preceq w_{k} \prec v_{j}$. Then

$$
[[\kappa]] \models\left(\exists w_{k}\right)\left(\forall v_{j}\right)\left[w_{i} \preceq w_{k} \prec v_{j} \vee \bigvee_{q} \eta_{q}\right]
$$


Let $c \in \kappa$ be a value for $w_{k}$ satisfying the last formula. Take a cofinal $Z$ such that $Z \cap[0, c]=\emptyset$. Then it is easy to verify that

$$
\left[\left[\kappa^{2}\right]\right] \models\left(\forall v_{j}\right)(\exists u)\left[\left(u=w_{i} \preceq c \prec v_{j} \vee \bigvee_{q} \eta_{q}\right) \wedge u \notin Z\right] .
$$

Indeed, for $v_{j}$ such that $c \prec v_{j}$, take $u=c$ for which the first clause holds and $c \notin X$. If $v_{j} \leq c$, then $v_{j}$ satisfies $\bigvee_{q} \eta_{q}$, where each $\eta_{q}$ contains the equation $u=v_{j}$. So if $u=v_{j}$, then $u \notin X$ and we are done. It follows that $\left[\left[\kappa^{2}\right]\right] \models(\exists X) \neg \phi(X)$.

(b) Let $w_{k} \prec u=w_{i} \preceq v_{j}$. Then

$$
[[\kappa]] \models\left(\exists w_{k}\right)\left(\forall v_{j}\right)\left[w_{k} \prec u=w_{i} \preceq v_{j} \vee \bigvee_{q} \eta_{q}\right]
$$

Let $c \in \kappa$ be a value for $w_{k}$ satisfying the last formula. Take a cofinal $Z$ such that $Z \cap[0, c+1]=\emptyset$ and $Z$ contains no two consecutive elements. Then it is easy to verify again that

$$
\left[\left[\kappa^{2}\right]\right] \models\left(\forall v_{j}\right)(\exists u)\left[\left(c \prec u=w_{i} \preceq v_{j} \vee \bigvee_{q} \eta_{q}\right) \wedge u \notin Z\right] .
$$

Indeed, for $v_{j} \leq c+1$, we pick $u=v_{j}$ which satisfies $\bigvee_{q} \eta_{q}$ and $v_{j} \notin Z$. For $v_{j}>c+1$, we pick any $u$ such $c<u<v_{j}$ and $u \notin Z$. So in any case the above holds. Thus again $\left[\left[\kappa^{2}\right]\right] \models(\exists X) \neg \phi(X)$.

(c) Let $w_{k} \prec v_{j} \preceq u=w_{i}$. Then

$$
[[\kappa]] \models\left(\exists w_{k}\right)\left(\forall v_{j}\right)\left[w_{k} \prec v_{j} \preceq u=w_{i} \vee \bigvee_{q} \eta_{q}\right] .
$$

Take $c$ for $w_{k}$ satisfying this formula and a cofinal $Z$ as in (b) above. Then we easily see again that

$$
\left[\left[\kappa^{2}\right]\right] \models\left(\forall v_{j}\right)(\exists u)\left[\left(c \prec v_{j} \preceq u=w_{i} \vee \bigvee_{q} \eta_{q}\right) \wedge u \notin Z\right]
$$

Hence $\left[\left[\kappa^{2}\right]\right] \mid=(\exists X) \neg \phi(X)$.

This exhausts the examination of Case 2. Note again that whenever in the above subcases $\left[\left[\kappa^{2}\right]\right] \models(\exists X) \neg \phi(X)$, then for every $X$, we can choose each $v_{j}$ arbitrarily high in $X$, unless the equation $u=v_{j}$ does not occur in $\theta$, or the choice of $v_{j}$ falsifies simultaneously all clauses containing $u=v_{j}$.

$\leftarrow$ CASE 3. Suppose Cases 1 and 2 are false. That means that there is no $(u=w)$-clause of $\theta$ in which for every pair of variables $w_{i}, v_{j}$, either $j<i$ (Case 1 ) or $i \leq j$ and $w_{i} \prec v_{j}$ is in the clause (Case 2). It follows that every $(u=w)$-clause of $\theta$ contains a formula of the form $v_{j} \prec w_{i}$ or $v_{j}=w_{i}$ such that $i \leq j$. Then we claim that $\left[\left[\kappa^{2}\right]\right] \models(\forall X) \phi(X)$. 
Proof. In order for $\left[\left[\kappa^{2}\right]\right] \models(\forall X) \phi(X)$ to hold, it suffices, for every $X$, to pick all $v_{j}$ so that (a) $v_{j} \in X$ and (b) these $v_{j}$ falsify all $(u=w)$ clauses. We do not need to bother about the truth or falsity of $(u=v)$ clauses, because for every such choice, they will define either $\emptyset$ or singletons contained in $X$, hence $R_{\theta}^{\kappa} \subseteq X$ is guaranteed. Now since each $(u=w)$ clause contains a formula of the form $v_{j} \prec w_{i}$ or $v_{j}=w_{i}$ with $i \leq j$ (i.e., $v_{j}$ depending on $w_{i}$ ) it suffices, given $X$ and $w_{i}$, to pick $v_{j} \in X$ such that $w_{i}<v_{j}$. The same dependent variable $v_{j}$ may dominate several $w_{i_{1}}, \ldots, w_{i_{m}}$. For every such finite sequence $w_{i_{1}}, \ldots, w_{i_{m}}$, we can pick $v_{j}>w_{i_{1}}, \ldots, w_{i_{m}}$ and $v_{j} \in X$. This is always possible because every $X$ is cofinal. (This is the first and last place where the cofinality of the sets $X$ is essential.)

Proof of the Main Theorem. Let

$$
\phi(X) \equiv(\bar{Q} \bar{w})(\forall u)(\theta(\bar{w}, u) \Rightarrow u \in X)
$$

be the canonical form of $\phi$, and let $\left[\left[\kappa^{2}\right]\right] \models(\forall X) \phi(X)$. Let also $\lambda$ be another uncountable cardinal. It suffices to show that $\left[\left[\lambda^{2}\right]\right]=(\forall X) \phi(X)$.

If $\bar{Q}=\bar{\forall}$, the claim follows from Lemma 2.2. So let $\bar{Q}$ contain existential quantifiers. In view of Lemmas 2.6 and 2.7, we may assume that $\theta=\bigvee_{p} \sigma_{p}$, where all $\sigma_{p}$ are equational. If $\theta$ contains only $(u=v)$-clauses, the theorem follows from Lemma 2.8. Hence let $\neg \theta$ contain $(u=w)$-clauses. If some $(u=w)$-clause has no $v$-variables, then by Lemma $2.9,\left[\left[\kappa^{2}\right]\right] \models(\exists X) \neg \phi(X)$, which contradicts our assumption. So suppose every $(u=w)$-clause of $\theta$ contains $v$-variables.

Then we come to Cases $1,2,3$ treated above. We see that $\left[\left[\kappa^{2}\right]\right] \models$ $(\forall X) \phi(X)$ is compatible only with Cases $1.1 .1 .1,1.1 .1 .2 .2,1.2 .1,1.2 .3 .1$, $2.1,2.2 .1$, and 3 . That is, every $(u=w)$-clause of $\theta$ satisfies the conditions of some of these cases. Moreover, as explained above, the choice of the value for the dependent variables $v_{j}$ can be done in such a way that all conditions are met simultaneously. Since these conditions are first-order, they guarantee the truth of $(\forall X) \phi(X)$ in any other structure $\left[\left[\lambda^{2}\right]\right]$ for uncountable $\lambda$. Therefore $\left[\left[\lambda^{2}\right]\right] \models(\forall X) \phi(X)$. This completes the proof of the Main Theorem.

3. $\exists_{1}^{1}$ positive formulas. Theorem 2.1 also holds for $\exists_{1}^{1}$ positive formulas, but the proof is much simpler. This is already suggested by the canonical form $(\bar{Q} \bar{w})\left(R_{\theta}^{\kappa}(\bar{w}) \subseteq X\right)$ of $\phi(X)$. Since the latter obviously holds in $\kappa$ for $X=\kappa$, it follows that $(\exists X) \phi(X)$ holds in $\kappa$ and similarly in every uncountable cardinal. However, this argument is fallacious, because as remarked after Lemma 1.3 of the first section, the canonical form holds only for $X$ different from the universal set. 
Proposition 3.1. For any uncountable cardinals $\kappa, \lambda$ and every normal positive $\phi(X)$,

$$
\left[\left[\kappa^{2}\right]\right] \models(\exists X) \phi(X) \Leftrightarrow\left[\left[\lambda^{2}\right]\right] \models(\exists X) \phi(X) .
$$

Proof. Let $\kappa, \phi(X)$ be given. Observe that

$$
\left[\left[\kappa^{2}\right]\right]=(\exists X) \phi(X) \Leftrightarrow\left[\left[\kappa^{2}\right]\right] \models \phi(\kappa)
$$

$\Leftarrow$ of (12) is straightforward, while $\Rightarrow$ follows from monotonicity of positive formulas: If $\phi(X)$ is true and $X \subseteq Y$, then $\phi(Y)$ is true. Now it is easy to check, by induction on the construction steps, that for every positive $\phi(X)$,

$$
\phi(\kappa) \equiv \chi \quad \text { or } \quad \phi(\kappa) \equiv \top
$$

where $\chi$ is a first-order formula. Combining (12) and (13), and the fact that $[[\kappa]] \equiv[[\lambda]]$, we have

$$
\begin{aligned}
{\left[\left[\kappa^{2}\right]\right]=(\exists X) \phi(X) } & \Leftrightarrow\left[\left[\kappa^{2}\right]\right] \models \phi(\kappa) \\
& \Leftrightarrow[[\kappa]] \mid=\chi(\text { or } \top) \Leftrightarrow[[\lambda]] \models \chi(\text { or } \top) \\
& \Leftrightarrow\left[\left[\lambda^{2}\right]\right]=\phi(\lambda) \Leftrightarrow\left[\left[\lambda^{2}\right]\right] \models(\exists X) \phi(X) .
\end{aligned}
$$

4. Some consequences of the Main Theorem. Given an infinite cardinal $\kappa$, observe that the principle $2^{\kappa}=\kappa^{+}$can be formulated as a $\forall_{1}^{1}$ positive monadic sentence holding in a certain subclass of $\mathcal{P}\left(2^{\kappa}\right)$. Namely,

$$
2^{\kappa}=\kappa^{+} \Leftrightarrow\left(2^{\kappa},\left[2^{\kappa}\right]^{>\kappa},<\right) \models(\forall X)(X \text { is cofinal }) .
$$

Indeed, if $2^{\kappa}=\kappa^{+}$then every $X \in\left[2^{\kappa}\right]^{>\kappa}$ has cardinality $\kappa^{+}$and therefore is cofinal in $\kappa^{+}$. If, conversely, $2^{\kappa}>\kappa^{+}$, then there is a set $X \in\left[2^{\kappa}\right]^{>\kappa}$, e.g. $\kappa^{+}$, which is not cofinal in $2^{\kappa}$.

" $X$ is cofinal" is the formula $(\forall x)(\exists y)(x \prec y \wedge y \in X)$, which is positive in $X$. Hence $(\forall X)\left(X\right.$ is cofinal) is $\forall_{1}^{1}$ positive monadic.

As $2^{\kappa}=\kappa^{+}$expresses a certain relationship between $2^{\kappa}$ and $\kappa$, one might reasonably argue that a wider class of such simple relationships could be common to all pairs of $\kappa, 2^{\kappa}$. A specific implementation of this general idea would be the claim that for all infinite $\kappa, \lambda$, the structures $\left(2^{\kappa},\left[2^{\kappa}\right]^{>\kappa},<\right)$ and $\left(2^{\lambda},\left[2^{\lambda}\right]^{>\lambda},<\right)$ satisfy the same $\forall_{1}^{1}$ positive monadic sentences. Call this principle the $\forall_{1}^{1}$ Positive Equity Principle $\left(\forall_{1}^{1}\right.$-PEP). That is,

$$
\left(\forall_{1}^{1} \text {-PEP }\right) \quad(\forall \kappa, \lambda \geq \omega)\left[\left(2^{\kappa},\left[2^{\kappa}\right]^{>\kappa},<\right) \underset{\text { pos }}{\forall_{1}^{1}}\left(2^{\lambda},\left[2^{\lambda}\right]^{>\lambda},<\right)\right] .
$$

The restriction to cofinal sets and to positive $\forall_{1}^{1}$ formulas makes the above principle minimalistic. Of course it would be desirable to have stronger versions for PEP, i.e., $\Gamma$-PEP for $\Gamma$ a class of formulas larger than $\forall_{1}^{1}$, provided its consistency with ZFC can be established. For the time being, the main theorem of the previous section implies the following.

THEOREM 4.1. $\mathrm{ZFC}+\forall_{1}^{1}$-PEP is consistent. 
Proof. It suffices to show that $\mathrm{ZFC}+\mathrm{GCH} \vdash \forall_{1}^{1}$-PEP. In the presence of $\mathrm{GCH}, \forall_{1}^{1}$-PEP becomes

$$
\left(\kappa^{+},\left[\kappa^{+}\right]^{>\kappa},<\right) \equiv_{\text {pos }}^{\forall 1}\left(\lambda^{+},\left[\lambda^{+}\right]^{>\lambda},<\right) .
$$

But

$$
\left[\kappa^{+}\right]^{>\kappa}=\left[\kappa^{+}\right]^{\kappa^{+}}=\operatorname{Cof}\left(\kappa^{+}\right)
$$

and similarly $\left[\lambda^{+}\right]^{>\lambda}=\operatorname{Cof}\left(\lambda^{+}\right)$. So the above reduces to

$$
\left.\left[\left[\left(\kappa^{+}\right)^{2}\right]\right] \equiv{ }_{\mathrm{pos}}^{\forall 1}\left[\left(\lambda^{+}\right)^{2}\right]\right],
$$

which follows from the Main Theorem.

Corollary 4.2. ZFC $+\forall_{1}^{1}$ PEP $\vdash(\forall \kappa, \lambda \geq \omega)\left(2^{\kappa}=\kappa^{+} \Leftrightarrow 2^{\lambda}=\lambda^{+}\right)$.

Proof. Immediate from (14).

Does $\forall_{1}^{1}$-PEP settle GCH? The answer is yes, unless a large cardinal assumption is made. Namely, suppose ZFC $+\forall_{1}^{1}$-PEP $\forall \mathrm{GCH}$. Then the consistency of $\forall_{1}^{1}$-PEP $+\neg \mathrm{GCH}$ implies the consistency of $(\forall \kappa)\left(2^{\kappa}>\kappa^{+}\right)$, and hence the consistency of $2^{\kappa}>\kappa^{+}$for a strong limit singular cardinal $\kappa$. But, by [4] (see also [5, Theorem 36.1]), this is equivalent to the consistency of the existence of a measurable cardinal with Mitchell order $\kappa^{++}$.

5. Separating $\omega_{m}$ from $\omega_{n}$. In this section we use (essentially) the formulas employed in $[1$, p. 19] to distinguish between the structures $\left(\omega_{1}, \operatorname{Cof}\left(\omega_{1}\right),<\right)$ and $\left(\omega_{2}, \operatorname{Cof}\left(\omega_{2}\right),<\right)$. The formula that makes this distinction is of the form $(\forall x)(\exists X) \psi$, where $\psi$ is normal but not positive.

Consider the formulas:

$$
\begin{aligned}
& \operatorname{succ}(x):(\exists y \prec x)(\forall z)(z \preceq y \vee x \preceq z), \\
& \operatorname{type}_{\omega}(X, x):(\forall y)(y \in X \& y \prec x \Rightarrow y=0 \vee \operatorname{succ}(y)), \\
& \operatorname{cof}(X, x): x=0 \vee \operatorname{succ}(x) \vee(\forall y \prec x)(\exists z \in X)(y \prec z \prec x), \\
& \operatorname{acc}_{\omega}(x):(\exists X)\left[\operatorname{cof}(X, x) \wedge \operatorname{type}_{\omega}(X, x)\right], \\
& \phi_{\omega_{1}}:(\forall x) \operatorname{acc}_{\omega}(x) .
\end{aligned}
$$

It is easy to check that $\phi_{\omega_{1}}$ says that every element has cofinality $\omega$, hence $\left(\omega_{1}, \mathcal{P}\left(\omega_{1}\right),<\right) \models \phi_{\omega_{1}}$, while $\left(\omega_{2}, \mathcal{P}\left(\omega_{2}\right),<\right) \models \neg \phi_{\omega_{1}}$. Now

$$
\phi_{\omega_{1}} \equiv(\forall x) \operatorname{acc}_{\omega}(x) \equiv(\forall x)(\exists X) \psi,
$$

where $\psi \equiv \operatorname{cof}(X, x) \& \operatorname{type}_{\omega}(X, x)$. Since type ${ }_{\omega}(X, x)$ is not positive, $\psi$ is also not positive.

Now, by the same token, the formula $(\forall x)(\exists X) \psi$ also distinguishes the structures $\left(\omega_{1}, \operatorname{Cof}\left(\omega_{1}\right),<\right)$ and $\left(\omega_{2}, \operatorname{Cof}\left(\omega_{2}\right),<\right)$, since the quantifier $\exists X$ can range only over cofinal sets. Analogous separation formulas can be found for the other cardinals $\omega_{m}, \omega_{n}, m, n \in \omega$. 


\section{References}

[1] J. R. Büchi and D. Siefkes, Decidable Theories II. The monadic second order theory of all countable ordinals, Lecture Notes in Math. 328, Springer, 1973.

[2] J. E. Doner, Definability in extended arithmetic of ordinal numbers, Dissertationes Math. (Rozprawy Mat.) 96 (1972).

[3] J. E. Doner, A. Mostowski and A. Tarski, The elementary theory of well-ordering-a metamathematical study, in: Logic Colloquium '77, A. Macintyre, L. Pacholski and J. Paris (eds.), North-Holland, 1977, 1-54.

[4] M. Gitik, The strength of the failure of the singular cardinal hypothesis, Ann. Pure Appl. Logic 51 (1991), 215-240.

[5] T. Jech, Set Theory, Springer, 2002.

[6] Y. Moschovakis, Elementary Induction on Abstract Structures, North-Holland, 1974.

Department of Mathematics

University of Thessaloniki

54124 Thessaloniki, Greece

E-mail: tzouvara@math.auth.gr

Received 15 July 2003;

in revised form 27 February 2004 